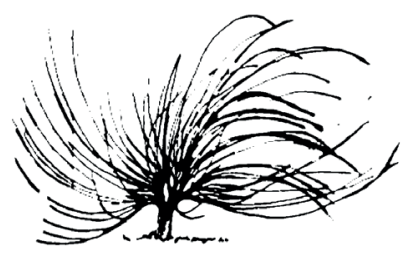

\title{
Transdisciplinariedad y pedagogía: apuntes para el debate sobre la (in)disciplina del pedagogo
}

\author{
Liliana Valladares ${ }^{1}$ \\ Universidad Nacional Autónoma de México \\ México \\ lvalladares@comunidad.unam.mx
}

\begin{abstract}
Resumen
En este ensayo, se reflexiona en torno al carácter transdisciplinario de la pedagogía y su relación con la identidad "híbrida" del pedagogo. Se revisan diferentes aproximaciones teóricas a la multi-, intery transdisciplinariedad en el estudio de la educación y se retoma, de forma sintética, la discusión alrededor del estatus epistemológico de la pedagogía. Se discute sobre si es posible comprender hoy la educación y el quehacer del pedagogo unidisciplinariamente; si puede la pedagogía considerarse transdisciplinaria, y si es la identidad híbrida del pedagogo una expresión del carácter transdisciplinario del estudio de la educación.
\end{abstract}

Palabras clave: transdisciplinariedad, pedagogía, identidad, disciplina.

\begin{abstract}
This essay wonders about the transdisciplinary nature of pedagogy and its relation to the "hybrid" identity of the pedagogue. Different theoretical approaches to multi, inter and transdisciplinarity in the study of
\end{abstract}

Recibido: 16 de mayo de 2019. Aprobado: 28 de octubre de 2019.

http://dx.doi.org/10.15359/rep.15-1.4

1 Profesora de carrera en la Facultad de Filosofía y Letras de la UNAM. Doctora en Filosofía de la Ciencia por la UNAM. Máster en Filosofía, Ciencia y Valores por la UPV-EHU. 
education are reviewed and the discussion about the epistemological status of pedagogy is synthetically recovered. It is discussed whether it is possible to understand education and the work of the pedagogue today from a unidisciplinary perspective; if pedagogy can be considered transdisciplinary, and if the hybrid identity of the pedagogue is an expression of the transdisciplinary character of the study of education.

Keywords: transdisciplinarity, pedagogy, identity, discipline.

\section{Introducción}

$\mathrm{E}$ n un par de trabajos publicados por Navarrete $(2007 ; 2008)$, dirigidos a comprender la identidad profesional del pedagogo universitario, la autora centra su mirada en la constitución de la pedagogía y de aquel como constructo híbrido. A partir de datos empíricos obtenidos en una investigación cualitativa realizada en dos universidades en México (Universidad Nacional Autónoma de México y Universidad Veracruzana), la autora alude a que el pedagogo denota una identidad mestiza, dado que ha recibido una formación disciplinar múltiple y heterogénea que es, a su vez, resultado de la constitución multidisciplinaria de la carrera de pedagogía.

De acuerdo con Navarrete:

La pedagogía se ha constituido como una disciplina híbrida, al igual que muchas otras, que abreva de distintas disciplinas (sociología, filosofía, psicología, antropología, etcétera)... La formación del pedagogo, al presentar este carácter de hibridación, dificulta hablar de la identidad profesional del pedagogo en términos unitarios y propios de la misma... pensar la formación del pedagogo sólo es posible como construcción articulatoria de múltiples saberes disciplinarios... (2007, p. 21).

La autora tiene razón en el hecho de que la trayectoria histórica de constitución híbrida de la disciplina pedagógica se ve reflejada en la identidad mestiza del pedagogo, pero resulta relevante preguntarse e indagar si esa hibridez responde también a un carácter transdisciplinario, 
transgresor, indisciplinado intrínseco de la propia pedagogía, que contrasta, por supuesto, con la fragmentación disciplinar a la que el pedagogo suele verse expuesto durante su formación.

El presente ensayo tiene como propósito introducir algunos elementos teóricos sobre las características de la transdisciplinariedad, como forma de producción de conocimiento, y discutir en torno a sus posibles correspondencias con ciertos rasgos de la pedagogía como campo de saber en el que confluyen múltiples miradas disciplinarias. Para ello, se discute alrededor de interrogantes tales como: ¿Es la identidad híbrida del pedagogo una expresión del carácter transdisciplinario propio del estudio de la educación? ¿Podemos distinguir a un pedagogo unidisciplinario y uno interdisciplinario de otro transdisciplinario? ¿Es la transdisciplinariedad un concepto que hoy permite replantearse la naturaleza epistemológica de la pedagogía y el quehacer del pedagogo?

\section{Desarrollo}

Cuando Ubal y Píriz (2009) se preguntan por el campo de conocimiento pedagógico, en un texto titulado ¿De qué hablamos cuando decimos pedagogía?, comprenden por este campo a la construcción discursiva (o teoría) ${ }^{2}$ sobre lo educativo que articula tanto procesos de circulación culturales como las relaciones humanas que los hacen posibles, al igual que los sentidos y prácticas que otorgan, reproducen, transforman los mencionados procesos y relaciones.

Con esta propuesta, Ubal y Píriz — como muchos historiadores y filósofos de la educación - pretenden trascender el sentido positivista-moderno de ciencia, para resaltar cómo la pedagogía conforma y posibilita una mirada particular sobre la educación, que conserva el carácter humanista de su etimología, sin reducirse a este. Vale la pena recordar que, si bien el término "pedagogía", como apunta Corominas (1987), deriva del griego paidagōgos que significa acompañante de niños (paidos = niño y ago $\bar{o}=$ yo conduzco), claramente el significado de esta disciplina, en la actualidad, va mucho más allá de la mera conducción o guía de los niños.

2 Se entiende por discurso un conjunto de prácticas, lingüísticas y no lingüísticas, portador de significados y constituido por un grupo de enunciados que, en su estructura semántica, poseen componentes epistémicos e ideológicos exhibidores de cierto grado de coherencia enunciativa (Ubal y Píriz, 2009). 
De la tradición alemana, a la pedagogía se le reconoce como disciplina, esto es, como un cuerpo sistemático de conocimientos que conforma y posibilita una mirada particular sobre la educación; aunque devela su carácter humanista, como lo expresa su etimología, también manifiesta el científico, como lo revelará su desarrollo histórico posterior. Hay dificultades para delimitar con exactitud las fronteras entre la educación y la pedagogía, mas se puede asumir que la producción de conocimiento (teórico, metodológico, axiológico) sobre la educación es el objeto de la pedagogía (Puiggrós y Marengo, 2013).

El hecho de que el fenómeno educativo, con toda su diversidad y complejidad, sea analizado por diversas ciencias (sociología, psicología, economía, política) que han permitido el avance del conocimiento sobre la educación, sus por qué, para qué y cómo, ha sido objeto de muchos y ricos debates en la reflexión pedagógica, así como ha dado pauta a la posibilidad de afirmar que la pedagogía, más que una disciplina unidisciplinaria es, sobre todo, un saber totalizador, unificador, que reconoce a otras ciencias de la educación, pero no se reduce a ellas. La pedagogía permite la coexistencia de los aspectos ontológicos, axiológicos, metodológicos, epistemológicos acerca de la educación (expresados en su fuerte base humanística y filosófica) y se fundamenta en ellos, pero también en el estudio científico de los factores sociales, culturales, históricos, económicos, biológicos, psicológicos que intervienen en el proceso educativo.

La pedagogía, en tanto disciplina autónoma sustentada en la construcción de una estructura teórico-metodológica propia de interpretación y generación del fenómeno educativo, puede reconocerse, de esta manera, como multi- o interdisciplinaria.

No obstante, el papel unificador que parece cumplir la pedagogía al respecto de las ciencias de la educación (ciencias cuya identidad no se reduce exclusivamente a lo educativo, pero que hoy resultan imprescindibles para el estudio de la educación) significa que esta genera conocimiento sobre la particularidad, no obstante, también sobre las articulaciones, relaciones o conjunciones posibles de los múltiples aspectos, facetas y dimensiones de la educación.

A partir de lo anterior, podemos pensar que la pedagogía responde más a un comportamiento transdisciplinar que de construcción multi- o interdisciplinaria sobre la educación. 
¿Qué diferencias hay entre uno y otro paradigma y cómo estas tenderían a expresarse en la identidad del pedagogo? ¿Es posible hablar de un pedagogo uni- e interdisciplinario y de uno transdiciplinario?

De acuerdo con Salmerón (1982, según se cita en Olivé, 2007) toda disciplina tiene tres rasgos característicos que la hacen especialmente apta para la enseñanza: 1) la síntesis de conocimientos en estructuras amplias y coherentes; 2) el análisis que simplifica los elementos cognoscitivos y los acomoda en esquemas comunes que facilitan su comprensión, y 3) un dinamismo que permite la incorporación de nuevos saberes.

Para Morin (1998), la disciplina es una categoría que organiza el conocimiento científico para instituir una división y especialización creciente del trabajo académico, que se corresponde con la diversidad de dominios y de objetos de estudio abarcados por las ciencias.

Sin embargo, Morin (1998) nos advierte cómo la conformación e institucionalización de las disciplinas, como principales modos de producción de la ciencia, a lo largo de su desarrollo histórico, aunque permiten organizar el conocimiento, entrañan, simultáneamente, un riesgo de hiperespecialización del investigador y uno de cosificación del objeto de estudio, del cual se tiende a olvidar que es extraído o construido: "el objeto de la disciplina será entonces percibido como una cosa en sí[...] El espíritu hiperdisciplinario va a devenir en un espíritu de propietario que prohíbe toda incursión extranjera en su parcela del saber" (Morin, 1998, p. 14).

En su sentido etimológico, la disciplina deriva de la palabra latina disciplina, que significa enseñanza y regla de vida, y que a la vez deriva de discipulus o "discípulo", quien recibe enseñanza de otro (Olivé, 2007).

También en su origen el término disciplina está asociado a un instrumento, ordinariamente de cáñamo, que sirve para azotar; esto es, como señala Morin (1998), el sentido en el que la disciplina se convierte, de manera distorsionada, en un medio de flagelación para aquellos quienes se arriesgan a atravesar las fronteras del dominio de ideas que un especialista disciplinario considera como de su propiedad. El debate sobre la disciplinariedad es, en consecuencia, muchas veces entendido como la disputa entre grupos de expertos en un cuerpo de conocimientos, por la propiedad de dicho campo de estudio (Palaiologou, 2010). 
Para Morin (1998), las disciplinas están justificadas intelectualmente a condición de que guarden un campo de visión que reconozca y conciba la existencia de las relaciones y solidaridades entre unas y otras, preservando la multiplicidad compleja de lo que es humano. Esto es, a condición de que sean ellas mismas indisciplinadas.

Con ejemplos concretos de la historia de la ciencia, Morin (1998) nos deja ver cómo han sido los actos de usurpación de un problema de una disciplina sobre otra, de circulación de conceptos, la fuente de explicaciones creativas que ha dado lugar a nuevas disciplinas de naturaleza híbrida.

Así, Morin (1998) describe cómo la mirada ingenua de personajes relevantes en el avance de las ciencias, como Alfred Wegener (quien desarrolló la teoría de la deriva continental) o de Charles Darwin (autor de la teoría de la evolución biológica), quienes se aproximaron con un ojo ajeno, amateur y nuevo a ciertas problemáticas, lograron proporcionar explicaciones creativas, que parecían invisibles al ojo de una disciplina particular de las ya establecidas en sus contextos sociohistóricos.

Estos actos de usurpación de un problema de una disciplina sobre otra, de circulación de conceptos, que ha dado lugar a nuevas disciplinas híbridas, permite a Morin (1998) introducir el concepto de intertranspolidisciplinariedad en el que "ciertas nociones circulan y, a menudo, atraviesan clandestinamente las fronteras sin ser detectadas por los aduaneros" (p. 11).

Las migraciones características de la intertranspolidisciplinariedad pueden entenderse si optamos, como Salmerón (1982, según se cita en Olivé, 2007), por pensar que las disciplinas no son fundamentalmente cuerpos de conocimiento, sino comunidades de investigadores que participan de una misma actitud. Esto conlleva pensar que la intertranspolidisciplinariedad alude a la interacción, convergencia o concurrencia de varias disciplinas y comunidades académicas, para la comprensión de un problema y, en muchas ocasiones, con el fin de orientar las acciones e intervenciones en el mundo, necesarias al tratar de resolverlo.

El debate sobre las diferentes formas progresivas de interacción entre disciplinas se remonta a los años 70 , cuando se advirtió que estas se relacionaban en grados que van desde una simple comunicación de ideas, hasta la transferencia e integración de conceptos, metodologías, datos y organización de la investigación y educación en muchos 
campos (Darbellay, 2015). Así, se hizo posible diferenciar entre la multi-, la inter- y la transdisciplina.

En una visión multidisciplinaria, un objeto de estudio dado o un problema conceptualizado requiere la solución propuesta de dos o más disciplinas que, conservando sus conceptos, métodos, prácticas, es decir, de forma compartimentalizada, aportarán conocimientos para comprender una cierta faceta o dimensión del fenómeno por estudiar; la interacción entre disciplinas se da más en forma aditiva, que integrativa (Darbellay, 2015).

En una visión interdisciplinaria, dos o más disciplinas interactuarán para permitir que la complejidad de un objeto de estudio sea descrita, analizada, comprendida mediante la colaboración, sinergia e integración disciplinaria, la cual puede consistir en la transferencia o apropiación de conceptos, valores, métodos de una tradición disciplinaria por otra. Esta hibridación puede dar lugar a nuevas disciplinas (interdisciplinarias) o a la creación de novedosos campos de investigación, pero no reconfigura los límites de las disciplinas de las que se abreva (Palaiologou, 2010).

La transdisciplina, en cambio, pone su acento en un proceso de conocimiento que trasciende los límites disciplinarios, reconfigurando parcelas disciplinarias en una perspectiva sistémica, global e integrada.

Un especialista uni- o interdisciplinario desarrollará una visión fragmentada de un problema basado en diferentes visiones disciplinarias, sin llegar a integrar una panorámica unificadora que trascienda los límites de estas visiones en nuevas epistemologías, ontologías, metodologías. El estudio de la educación requiere, sin duda, el desarrollo de una capacidad "trans-" que supere e integre las fronteras disciplinarias, unificando conocimiento en un vocabulario común, acorde con la complejidad de problemáticas educativas de la sociedad actúa. Esta capacidad "trans-" es una creativa, que resulta del encuentro de diferentes disciplinas y que va más allá de ellas para consolidar conocimiento sobre lo educativo. Considerando su enorme complejidad y dinamismo, la transdisciplina es el concepto que mejor caracteriza el quehacer de quien se especializa en estudiar la educación (Palaiologou, 2010).

Son dos las principales aproximaciones al entendimiento de lo que es hoy la transdisciplina (Augsburg, 2014). Por un lado, el grupo de Gibbons, Limoges y Nowotny (1997), identificó el llamado Modo 2 de conocimiento para explicar la forma en que se produce saber 
en un marco de globalización, ciencia posnormal y tecnologías de la información.

Según Gibbons et al. (1997), son cuatro las principales características de la transdisciplina:

1. Genera nuevos marcos teórico-metodológicos que no se pueden reducir a los disciplinares, y lo hace para dar solución a problemas específicos.

2. El conocimiento generado no se identifica, por lo tanto, con alguna disciplina particular, sino que comprende la conformación de nuevas estructuras teóricas y metodológicas.

3. La comunicación y difusión del conocimiento generado no se queda solo en el nivel de publicaciones especializadas, sino que se pone a disposición y resulta accesible para los diferentes involucrados, en torno a un contexto de aplicación determinado. De hecho, no solamente los expertos científicos son quienes participan en el proceso de generación de conocimiento transdisciplinario, este resulta de la interacción y negociación de todos los involucrados en el problema (expertos y no-expertos).

4. En el conocimiento transdisciplinario, tiene cabida una gran variedad de posibles aplicaciones, muchas de las cuales trascienden al contexto mismo en el que inicialmente fue creado.

En suma, para el grupo de Gibbons et al. (1997), la transdisciplina se distingue de la multi- y la interdisciplina porque, además de utilizar conceptos y métodos que provienen de diferentes disciplinas y de forjar nuevos conceptos y métodos que no se identifican con alguna disciplina en particular, esta se constituye frente a problemas específicos, los cuales permiten que grupos heterogéneos de actores transdisciplinarios (incluyendo a los usuarios, implicados o interesados en algún problema particular o, en suma, a la sociedad civil) puedan ser los constructores de conceptos novedosos y métodos adecuados para entender y atender tales inconvenientes específicos aquejantes, con el fin de resolverlos.

Por otro lado, destaca el trabajo de Nicolescu (1999), cuya forma de entender la transdisciplinariedad estuvo ligada a considerar que la complejidad es una característica fundamental de la realidad y que acceder a ella implica no solamente la coordinación de equipos transdisciplinarios (heterogéneos), sino el desarrollo de actitudes 
transdisciplinarias de quienes participan en procesos de generación de conocimiento (Augsburg, 2014).

Nicolescu (1999) consideraba que la transdisciplinariedad es una metodología para crear conocimiento nuevo sobre lo que está entre, a través y más allá de las disciplinas, la cual asume que todo es dependiente de todo, que nada está separado y que todo está interrelacionado (Neuhauser y Pohl, 2015).

La transdisciplinariedad, en este sentido, representa un desafío para el pensamiento disciplinar y sus formas establecidas institucionalmente, pues busca configurar nuevas aproximaciones a partir de la interacción, la interperspectividad y la combinación disciplinaria, yendo más allá de las disciplinas (es decir, que se nutre de fuentes científicas y no-científicas), en lo que Klein (2015) llama "el discurso de la transgresión".

Aunque se le atribuye a Jean Piaget la introducción del término transdisciplina, en 1972 fue Erich Jantsch quien asoció la transdisciplinariedad a una propuesta de reformar la educación superior, no en departamentos disciplinarios, sino en sistemas orientados por metas para la resolución de problemas socialmente relevantes, lo que — según pensaba Nicolescu (1999) — reduciría la brecha entre la academia y la sociedad (Neuhauser y Pohl, 2015).

El discurso de la transgresión unifica conocimiento en un intento por superar el entendimiento fragmentado que en ocasiones se construye acerca del mundo. Al buscar la elaboración de representaciones relacionales que reflejen la complejidad de los campos y problemáticas educativas en sus muchos contextos y considerando distintas voces (profesores, padres y madres de familia, estudiantes, funcionarios de la educación, investigadores educativos, entre otros), la pedagogía transgrede institucionalmente el modus operandi de la disciplinariedad (Augsburg, 2014).

A ello se suman dos características de la pedagogía que sugieren su constitución como campo de conocimiento transdisciplinario: I) su ubicación particular en el mapa disciplinario que autores como Piaget et al. (1973) y Max-Neef (2005) han elaborado; II) la naturaleza de la educación como problema complejo y multifacético, inaprehensible por una sola disciplina no-transdisciplinaria. Veamos.

Cuando se revisa el mapa disciplinar de la pedagogía descrito por Velázquez (2005), es posible observar cómo algunas de las características definitorias de la transdisciplina, principalmente aquellas 
desarrolladas por Max-Neef (2005), aplican a la pedagogía. Esto hace pensar que este campo de conocimiento, atravesado por muchas dimensiones y niveles, no puede fácilmente ubicarse en los mapas clasificatorios tradicionales de las disciplinas, más que como una disciplina de naturaleza transdisciplinaria.

De acuerdo con Velázquez (2005), cuyos planteamientos se seguirán muy de cerca en los siguientes párrafos, pues permiten comprender la naturaleza compleja del estudio de la educación, el modelo piagetiano de las ciencias humanas resulta esclarecedor para entender dónde y cómo ubicar la pedagogía en el mapa disciplinar de las ciencias.

Según Velázquez (2005), en el modelo piagetiano de clasificación de las ciencias humanas, la pedagogía se ubica en una posición intermedia y pluridireccional, subsidiaria y a la vez complementaria de las ciencias humanas, pues en el estudio de la educación se manifiesta, simultánea y casi invariablemente, la participación de las dimensiones "nomotéticas, históricas, jurídicas, normativas y reflexivo-filosóficas” (p. 7).

Estas dimensiones que atraviesan lo educativo se corresponden, a su vez, con la clasificación de las ciencias humanas que hacen Piaget et al. (1973). Esta categorización consiste en cuatro tipos de ciencias, a saber (Velázquez, 2005): a) nomotéticas (establecen leyes, relaciones cualitativas y cuantitativas entre elementos empíricos; elaboración de hechos y conceptos mediante metodologías prioritariamente analítico-experimentales); b) históricas (buscan reconstruir y comprender, en el eje del tiempo, las manifestaciones de la vida social, así como hechos, situaciones y sistemas socioculturales); c) jurídicas (estudian el "deber ser" y la vinculación entre las atribuciones y obligaciones prescritas en normativas), y d) disciplinas filosóficas (indagan y formulan concepciones del mundo, mediante la reflexión totalizadora de la realidad y del resto de las ciencias).

Cuando Velázquez (2005) trata de ubicar la pedagogía en la clasificación previa, surge una característica peculiar que dificulta dicha tarea, pero que al mismo tiempo proporciona información sobre la naturaleza epistemológica singular de esta disciplina: el fenómeno educativo - su objeto de estudio - es subsidiario de todas estas diversas ciencias. La aproximación que cada disciplina aporta en torno a este objeto, de forma independiente y aislada, excede la posibilidad de construir un discurso comprehensivo de este fenómeno, el cual sea capaz de abarcar todos sus ángulos y delinear cursos de acción que permitan enfrentar 
y transformar la realidad educativa, siempre diversa, contradictoria y cambiante, en cuanto problema complejo de nuestras sociedades.

Como apunta Velázquez (2005), el objeto de estudio de la pedagogía es un fenómeno multifacético que emerge de la superposición de una diversidad de componentes, resultado de la intervención de más de una disciplina. Conceptos y categorías pedagógicas, por ejemplo "el sujeto del aprendizaje, la formación docente, la relación docente-alumno-conocimiento, la práctica docente, las organizaciones educativas, los métodos, técnicas y estrategias de intervención docente, entre otros" (Velázquez, 2005, p. 12), han sido producto de la interacción disciplinaria. Profundizar en su conocimiento, en aras de su transformación práctica, requiere igualmente visiones inter- $\mathrm{y}$ transdisciplinarias características de la construcción del discurso pedagógico.

Puiggrós y Marengo (2013) destacan cómo, para la construcción del conocimiento sistemático acerca de la educación, se ha requerido simultáneamente ciencias diversas que, sin tener como objeto de estudio exclusivamente lo educativo, cuentan con un aparato teórico-metodológico para el abordaje de este fenómeno. Sin embargo, es importante destacar que su aportación no consiste solo en vincular el conocimiento generado por estas otras ciencias en los problemas de la práctica educativa, sino en producir, en conjunto, nuevo conocimiento, de naturaleza pedagógica, emergente de la articulación entre dichas disciplinas (esto es, un metalenguaje que incluye distintos niveles de la realidad educativa).

En sus muchas manifestaciones y formas (como práctica, proceso, acción, institución), la educación es así un objeto de estudio transdisciplinario. Su configuración actual, como problema social relevante, demanda el trabajo de redes epistémicas compuestas por actores heterogéneos académicos y no académicos (que incluyen no solo a los pedagogos, sino a agentes de la comunidad educativa - profesores, estudiantes, padres y madres de familia, autoridades educativas - y otros expertos de disciplinas, entre cualquiera de los cuatro tipos de la taxonomía piagetiana), un rasgo propio de la transdisciplina.

La educación, como se ha argumentado en Valladares (2017), puede pensarse como un complejo interdependiente de, al menos, cinco tipos de prácticas educativas: i) de aprendizaje; ii) de enseñanza; iii) de formación docente o aprendizaje profesional; iv) de dirección y gestión de la educación, y v) de investigación educativa. En estas prácticas, simultáneamente: a) ocurre el proceso educativo; b) se deja 
registro sociohistórico-material de la educación y del cambio educativo; c) se genera conocimiento (pedagógico) sobre sí mismas —en tanto prácticas-y sobre el resto de las prácticas con las que se imbrican. Por consiguiente, para explicar, interpretar, decidir, prescribir y ordenar la educación, en cuanto objeto de la pedagogía, no se puede obviar la naturaleza compleja y transdisciplinaria de la ecología de prácticas que configura su objeto de estudio.

En tal sentido, un pedagogo puede conocer y aplicar tanto métodos como conceptos de, por ejemplo, la sociología para entender lo educativo, pero la explicación cabal de su objeto de estudio no podría reducirse a solo el abordaje de los aspectos sociológicos de la educación. Lo mismo podría poner en funcionamiento conceptos y métodos de la historia, con el fin de comprender un problema, hecho, institución o práctica educativa, pero su entendimiento de lo educativo, para constituirse como auténticamente pedagógico, no podría de reducirse a solo lo histórico, sino que habría de considerar las otras dimensiones de la educación, como fenómeno complejo en el que se mezclan aspectos filosóficos, económicos, culturales, antropológicos, políticos, sociales, éticos, psicológicos, entre muchos otros.

En el estudio de las prácticas educativas, según el modelo piagetiano (Piaget et al., 1973; Piaget y Desmet, 1992), recuperado por Velázquez (2005), convergen, de este modo, las dimensiones psicológica y social, teórica y práctica, explicativa y normativa, necesarias para investigar tanto los procesos intrasubjetivos de los humanos (por ejemplo, la construcción genética del psiquismo, el aprendizaje y las vinculaciones con los procedimientos constitutivos del sujeto, el desarrollo de la identidad, entre muchos otros) como los procesos intersubjetivos que abarcan. Lo anterior, tal como señalan Puiggrós y Marengo (2013), desde la relación entre un educador y un educando hasta la constitución, reproducción y transformación de formaciones sociales y del plano institucional (socialización, escolarización, relación entre la población y el Estado como educador, entre otros).

En la dimensión histórica, la pedagogía buscará comprender los hechos educativos, el desarrollo de las ideas pedagógicas, las prácticas, así como las instituciones, estructuras y sistemas de la educación, más su constitución y funcionamiento actual, como resultados históricos de la vinculación de factores psicológicos, sociales, culturales, económicos, religiosos. Como describen Puiggrós y Marengo (2013), 
la educación debe ser estudiada y definida en cada momento histórico, como expresión de un presente social situado, pero también como opciones de futuro.

En su dimensión normativa, "singularmente más acentuada que en otras disciplinas humanas, pues su objeto es el proceso de la configuración de la realidad humana" (Velázquez, 2005, p. 8), la pedagogía permitirá indagar las prácticas educativas y sus normativas diversas, en cuanto modelos ideales de humanos, sociedad y educación.

En el estudio de la normatividad educativa se entrecruzan, paralelamente, las dimensiones nomotéticas e históricas del saber pedagógico, por un lado, contenidas en las aportaciones del conocimiento científico que producen las ciencias nomotéticas y las históricas sobre los hechos y fenómenos educativos, al igual que sobre sus bases empíricas y procesos constitutivos; por otro, la dimensión filosófica-reflexiva, derivada del campo de la filosofía y que permite el estudio de los aspectos ideológicos, axiológicos y de crítica epistemológica de lo educativo (Velázquez, 2005).

De manera específica, y siguiendo a Velázquez (2005), en esta dimensión filosófica de la pedagogía, se pueden distinguir tres tipos de aproximaciones al objeto de estudio: a) una fenomenológica a los hechos y procesos educativos, enfocada en la comprensión —no en la explicación - de la educación y que incluye diversas metodologías comprensivo-interpretativas que abarcan "desde la intuición, introspección a la deducción, experiencia personal, sentimiento, contraste de opiniones" (p. 8); b) una epistemológica, encauzada al análisis crítico de los fundamentos de la estructura científica del discurso educativo, y c) una axiológica, ética y teleológica, propia de la dimensión normativa del estudio de la educación, y que permite reconocer los elementos tanto ideológicos como valorativos que participan en toda práctica educativa.

El estudio de la educación constituye, de este modo:

Un espacio científico donde confluyen las más diversas aportaciones, desde las ciencias y disciplinas que se ocupan de las distintas manifestaciones de la realidad humana a las ciencias formales que ofrecen modelos y técnicas de observación, medición, análisis, información, deducción, instrumentos, todos ellos de investigación, de verificación experimental de la teoría y modelos de 
coordinación, integración, combinación y estructuración de las diversas informaciones recibidas (Velázquez, 2005, p. 9).

Para complejizar este esquema y, a modo de dar cuenta de cómo la naturaleza de la pedagogía escapa a los marcos disciplinarios con los que comúnmente se pueden entender otras ciencias de carácter mayormente unidisciplinario, cabe destacar, además, que, en el modelo relacional entre ciencias nomotéticas, históricas, jurídicas y filosóficas desarrollado por Piaget y Desmet (1992), el estudio de lo educativo puede también ser visto desde dos perspectivas vinculadas dialécticamente (Velázquez, 2005): i) una sincrónica, la cual incluye los componentes básicos para el estudio de la educación, como las ciencias y disciplinas humanas que, desde sus respectivos campos de conocimiento, aportan conceptos, teorías, modelos formales, técnicas empíricas en torno a lo educativo; ii) una diacrónica, que explica el funcionamiento de dicha estructura y en la que se manifiestan las dimensiones teórica, proyectiva y práctica, constitutivas del eje de la educación como campo de conocimiento. Estas tres nuevas dimensiones que conforman otro ángulo de la complejidad del campo pedagógico se relacionan entre sí de manera circular - es decir, que la modificación continua de una de estas dimensiones implica, necesariamente, cambios en las otras-.

De acuerdo con Velázquez (2005), dichas dimensiones caracterizan a la pedagogía como un sistema abierto de comunicación del que emerge conocimiento de la educación, el cual no podría producirse desde solo una dimensión o únicamente un tipo de disciplina, sino de la articulación de sus elementos diversos y también de sus tensiones.

Así, la dimensión teórica tiene la función de elaborar teorías específicas sobre la estructura y el desarrollo tanto de los fenómenos como de los procesos educativos y, para ello, recupera organiza, integra y sistematiza las aportaciones que emergen sobre la educación, desde la mirada de distintas disciplinas. Por su parte, la dimensión proyectiva permite construir y diseñar modelos formales y programas complejos de investigación o aplicación educativas, para su futura implementación en ambientes educativos diversos. Finalmente, la dimensión práctica integra el conjunto de métodos, instrumentos, técnicas, procedimientos concretos que se ponen en acción, mediante el despliegue de un proyecto o programa educativo particular, haciendo uso del conocimiento generado por las otras dimensiones (Velázquez, 2005). 
Particularmente, afirma Velázquez (2005), es la dimensión proyectiva la que le otorga un papel relevante a la pedagogía como producto y proceso de conformación de lo intersubjetivo y lo intrasubjetivo, pues interviene en la conformación del pensamiento acerca de la educación, al mismo tiempo que la construye. Es decir, la educación, su objeto de estudio, cambia por acción y efecto de la influencia de las formaciones culturales y así, simultáneamente, estructura y es estructurada por el discurso pedagógico y viceversa.

Ahora bien, la coexistencia de estas dimensiones del modelo piagetiano, las cuales permiten cartografiar el quehacer de la pedagogía como transversal a los cuatro tipos de disciplinas, está también presente en Larroyo (1949), quien organiza el campo de la pedagogía en: 1) ontología de la educación (estudio del hecho de la educación, sus tipos, grados y leyes); 2) axiología y teleología de la educación (estudio de los fines de la educación y la formación de valores en los seres humanos); 3) didáctica (estudio de los métodos y tareas de enseñanza), y 4) organización y administración educativas (estudio de la organización y administración del proceso pedagógico). Similarmente, Luzuriaga (1973) distingue entre: a) pedagogía descriptiva: enfocada en los hechos, factores e influencias de la realidad educativa; b) pedagogía normativa: centrada en los fines e ideales de la educación, y c) pedagogía tecnológica: focalizada en los métodos, organización e instituciones de educación (De Battisti, 2011).

El hecho de pensar a la pedagogía como ciencia de carácter totalizador y unificadora de estas dimensiones descriptivas, explicativas, praxeológicas, normativas (De Battisti, 2011) encuentra una clara correspondencia con la manera en que Max-Neef (2005) distingue a los campos transdisciplinarios de conocimiento. De acuerdo con este autor, las disciplinas pueden organizarse y distribuirse en un mapa con forma de pirámide. En la base, encontramos el primer nivel jerárquico -el empírico- - y en la cúspide, el nivel valórico; entre ellos se encuentran el nivel pragmático y el normativo. Cada uno se define por la búsqueda heurística de respuestas a un tipo de preguntas clave (Max-Neef, 2005):

1. El nivel empírico corresponde a disciplinas que se preguntan sobre lo que existe en el mundo tanto social como natural y buscan respuestas acerca del comportamiento de estos hechos, fenómenos, situaciones; aquí se incluyen disciplinas que 
describen el mundo como es y que construyen teorías, al igual que leyes físicas de la naturaleza, así como principios, modelos, enfoques que gobiernan la vida en común y las sociedades. En este nivel, se instalan áreas como, por ejemplo, física, química, biología, psicología, sociología, economía, entre otras. Además, coincide, en gran medida, con las ciencias nomotéticas de Piaget.

2. El nivel pragmático incluye disciplinas que se cuestionan sobre lo que somos capaces de hacer, ofreciendo orientaciones propositivas o tecnológicas, como sucede, por ejemplo, en la arquitectura, la medicina, las ingenierías, la agronomía, entre otras.

3. El nivel normativo alude a disciplinas que indagan sobre qué es lo que queremos hacer, ofreciendo orientaciones formales; por ejemplo, el diseño ambiental, la política, el derecho. En este nivel, podríamos ubicar las ciencias jurídicas/normativas de Piaget.

4. El nivel moral comprende disciplinas que se preguntan sobre qué debemos hacer o cómo deberíamos hacer lo que queremos, buscando criterios epistemológicos y valóricos legítimos. En este nivel, pueden incluirse, por ejemplo, disciplinas como la filosofía, la ética, la teología, y claramente observamos una correspondencia de este con las disciplinas filosóficas de Piaget.

En términos generales, en la integración e interacción disciplinaria que deriva del mapa disciplinario de Max-Neef (2005), las disciplinas transitan desde un nivel "empírico" hacia uno "propositivo", para continuar con lo "normativo" y terminar en lo "valórico".

Ahora bien, para Max-Neef (2005), la interdisciplinariedad es una forma de organización disciplinaria que, en la pirámide expuesta, comprende dos de los cuatro niveles jerárquicos descritos. Es decir, un campo interdisciplinario será aquel en el que hay cooperación y se establecen relaciones entre disciplinas diversas pertenecientes a dos niveles jerárquicos de la pirámide. Con esta implicación de doble nivel, el resultado es que, en el desarrollo de nuevos campos de conocimiento, producto de la imbricación de niveles, se introduce un nuevo sentido de propósito a un grupo de disciplinas originalmente ubicadas en un nivel inferior, transitando al nivel jerárquico inmediatamente superior. Así, por ejemplo, la biotecnología, en su carácter interdisciplinario, sería el 
resultado de atribuir un nivel pragmático/tecnológico a una disciplina de nivel empírico como es la biología.

En contraste, en la transdisciplinariedad convergen disciplinas de los cuatro niveles jerárquicos de la pirámide, lo que implica una coordinación entre todos los niveles de esta: "Cualquiera de las múltiples relaciones verticales posibles entre los cuatro niveles, definen una acción transdisciplinaria" (Max-Neef, 2005, p. 9).

Así pues, cuando la pedagogía se distingue de otras disciplinas y se define por su carácter empírico, pragmático, tecnológico, pero, al mismo tiempo, por su carácter propositivo, normativo y valorativo, se alude, de alguna manera, a su transdisciplinariedad constitutiva. Es una disciplina, en cuanto a que produce conocimiento particular en un espacio académico en apariencia delimitado (Puiggrós y Marengo, 2013), pero es una disciplina transdisciplinaria, cuando implica un saber que emana del entrecruce de disciplinas situadas en los diferentes niveles jerárquicos propuestos por Max-Neef (2005).

$\mathrm{Si}$ es posible hablar de un pedagogo unidisciplinario, multi- y transdisciplinario, la diferencia estaría dada, entonces, por el número de niveles jerárquicos que involucra en la construcción del discurso pedagógico; si solo abarca aspectos empíricos, sin considerar las consecuencias, las implicaciones, los alcances y limitaciones de sus aportaciones en los aspectos pragmáticos, normativos y valorativos de la educación, se estará ante un pedagogo unidisciplinario al que puede criticársele su orientación reduccionista, instrumental o acotada del proceso educativo. Este profesional contará con algunos elementos para aproximarse al estudio de la educación, pero su visión sobre ella quizá no sea del todo "pedagógica", en el sentido de la carencia de una panorámica comprehensiva sobre la multidimensionalidad de la educación. La posibilidad de hablar de estos tres tipos de pedagogos y de su coexistencia queda como pregunta abierta para futuros debates.

\section{Reflexiones finales}

En este ensayo, la intención ha sido poner sobre la mesa de debate qué tanto la noción de transdisciplinariedad nos invita a reflexionar sobre la formación del pedagogo y su identidad como un profesional que sabe un poco, o mucho, de todo para interpretar y comprender (integrada o fragmentariamente) el fenómeno educativo. 
En el campo pedagógico actual, autores como Díaz (2019) han criticado la mayor dedicación al estudio de los aspectos instrumentales, metodológicos, técnicos de la pedagogía y el poco interés en indagar en sus aspectos relacionales con respecto a otras categorías (entre las que señala, las tecnologías, la globalización, las relaciones de clase, el poder, el control, el lenguaje) necesarias para entender el papel del discurso pedagógico como dispositivo socializante, político, histórico, cultural. Frente a lo que llama el "instrumentalismo metodológico imperante" (Díaz, 2019, p. 26), este autor enfatiza que la pedagogía es más que una matriz social, técnicamente analizable y que pensar esta disciplina implica analizarla relacionalmente, en una categoría plural que es producto y productora de una diversidad discursiva sobre lo educativo.

En sentido similar, Puiggrós y Marengo (2013) advierten que la pedagogía no es solo la racionalidad que subyace a la intervención técnica de la enseñanza, sino "un campo de articulación de conocimientos, saberes y experiencias, sistematizado con metodologías diversas, generalmente asociadas a la producción filosófica, de las ciencias sociales y de la psicología" (p. 19).

No obstante, hoy parece primar un "estrecho código disciplinar", como lo llama Carbonell (2015, p. 211), presente en propuestas formativas instrumentales que usualmente devienen en una disminución de aptitudes para contextualizar saberes y captar lo que está tejido conjuntamente, las cuales propician la mera acumulación y sobrecarga de información que, como afirma este autor, no se estructura ni transforma en conocimiento sobre algún proceso o fenómeno del mundo. Según Carbonell (2015), la reducción del conocimiento del todo al conocimiento de las partes

supone fraccionar los problemas y reducir lo complejo a lo simple, imponiéndose el paradigma artificioso del determinismo mecanicista; la organización y transmisión del saber troceado dificulta las visiones holísticas y sistémicas que tanto ayudan a una mejor comprensión del mundo (p. 211).

Frente a esto, lo que hoy se sabe de la pedagogía, afirma Díaz (2019), ha sido resultado de un cúmulo indefinido de distintas posturas teóricas, metodológicas, ideológicas, éticas, estéticas, políticas, religiosas, de autores de diferentes épocas y campos de conocimiento 
(filosófico, psicológico, antropológico, sociológico, entre otros), quienes han coadyuvado a configurar el campo discursivo pedagógico. La pedagogía es, así, un campo heterogéneo, históricamente situado, pero de vigencia transhistórica (al incluir lo retrospectivo, lo presente y lo prospectivo), en el que intervienen diferentes tipos de sujetos que elaboran el discurso pedagógico y se ocupan del hecho educativo y de sus consecuencias (Puiggrós y Marengo, 2013), donde confluyen normas, prácticas, saberes, valores, "de límites inciertos" (Díaz, 2019, p. 17).

La comprensión de la identidad profesional del pedagogo del siglo XXI, como el producto híbrido al que se refiere Navarrete (2007), pasa por cuestionarnos si quienes se forman en la disciplina pedagógica son mayormente pedagogos que sostienen una visión uni-, multi- o interdisciplinar de la educación o si, en su lugar, se trata de profesionales transdisciplinarios que aprovechan y trascienden la estructura de las disciplinas, para configurar una visión integrada de la educación, lo suficientemente compleja como para abordar las problemáticas pluridimensionales asociadas a lo educativo, con creatividad y compromiso social e histórico.

El asumir una perspectiva transdisciplinaria de la pedagogía en los planes y programas de estudio de esta disciplina, permitirá lograr que los pedagogos del siglo XXI, en su desempeño académico y profesional, atraviesen los límites disciplinarios de las muchas asignaturas que cursarán en su formación, así como las fronteras de las múltiples disciplinas que requieren conocer para comprender los distintos campos educativos en sus diferentes contextos. Solo así estarán en condiciones de proponer soluciones innovadoras de mejora educativa desde la interperspectividad que les da la pedagogía, y únicamente de tal modo podrán superar la tendencia a reducir su hibridez a un mero reflejo de una simple fragmentación disciplinaria reforzada, en ocasiones, en su proceso formativo.

Para Augsburg (2014), la transdisciplinariedad presupone una ética individual y un deseo de contribuir al bien común que puede cultivarse. Volverse transdisciplinario, dice esta autora, supone actitudes como la adaptabilidad, flexibilidad, creatividad, apertura y un profundo interés en construir puentes y aventurarse al abordaje de un objeto de estudio o problema desde la (in)disciplina, a modo de configurar un punto de vista integrador y diferente que se apoya en muchas disciplinas, sin reducirse a ellas. 
Una identidad transdisciplinaria hará que quienes participan en la generación de conocimiento sobre una problemática particular sean coproductores de formas híbridas de conocimiento, dando cabida a múltiples voces sobre la educación.

Frente a la complejidad de las sociedades actuales, hoy es fundamental la formación de pedagogos transdisciplinarios que sean capaces de investigar lo educativo desde la especificidad pedagógica, con un sólido conocimiento humanístico, encadenado a la filosofía, esa que les permita comprender, en un sentido amplio, los componentes de la educación (sus fines, principios, valores, contextos) y sus relaciones, para intervenir argumentativa o propositivamente en el ámbito educativo.

Este carácter transdisciplinario, constante transgresor de las fronteras disciplinarias, si ha de manifestarse en la identidad del pedagogo, significaría que este, a través de una hibridación creativa de disciplinas, domina el saber pedagógico, es decir, cuenta con la fundamentación teórica, tecnológica y axiológica dirigida a explicar, interpretar, decidir, prescribir y ordenar la práctica de la educación (García-Aretio, 2009), desde su amplia comprensión.

\section{Referencias}

Augsburg, T. (2014). Becoming Transdisciplinary: The Emergence of the Transdisciplinary Individual. World Futures, 70, 233-247. Recuperado de https://www.tandfonline.com/doi/abs/10.1080/0 2604027.2014.934639

Carbonell, J. (2015). Pedagogías del siglo XXI. Barcelona: Octaedro. Corominas, J. (1987). Breve Diccionario Etimológico de la Lengua Castellana. Madrid: Gredos.

Darbellay, F. (2015). Rethinking inter- and transdisciplinarity: Undisciplined knowledge and the emergence of a new thought style. Futures, 65, 163-174. Recuperado de https://www.sciencedirect. com/science/article/pii/S0016328714001700

De Battisti, P. (2011). Clasificaciones de la pedagogía general y pedagogías especificas: un análisis de las demarcaciones efectuadas por especialistas del campo pedagógico. Recuperado de http://www. memoria.fahce.unlp.edu.ar/trab_eventos/ev.960/ev.960.pdf

Díaz, M. (2019). ¿Qué es eso que se llama pedagogía? Pedagogía y Saberes, 50, 11-28. Recuperado de https://doi.org/10.17227/pys. num50-9485 
García-Aretio, L. (2009). Claves para la educación. Actores, agentes y escenarios en la sociedad actual. Madrid: Narcea.

Gibbons, M.; Limoges, C.; Nowotny, H.; Schwartzman, S.; Scott, P. y $\mathrm{M}$. Trow. La nueva producción del conocimiento. La dinámica de la ciencia y la investigación en las sociedades contemporáneas. Barcelona: Ediciones Pomares.

Klein, J. (2015). Reprint of 'Discourses of transdisciplinarity: Looking back to the future'.Futures, 65, 10-16. Recuperado dehttps://www. sciencedirect.com/science/article/pii/S001632871500004X

Larroyo, F. (1949). La ciencia de la educación. México: Porrúa.

Luzuriaga, L. (1973). Historia de la educación y de la pedagogía. Buenos Aires: Losada.

Max-Neef, M. (2005). Foundations of transdisciplinarity. Ecological Economics, 53, 5-16. Recuperado de https://doi.org/10.1016/j. ecolecon.2005.01.014

Morin, E. (1998). Sobre la interdisciplinariedad. Recuperado de http:// www.edgarmorin.org/images/publicaciones/edgar-morin-sobre-la-interdisciplinariedad.pdf

Navarrete, Z. (2007). Identidad profesional del pedagogo universitario. Pampedia, 3, 12-21. Recuperado de http://www.scielo.org.mx/ scielo.php?script $=$ sci_arttext\&pid $=$ S1405-66662008000100007

Navarrete, Z. (2008). Proceso de construcción identitaria del pedagogo universitario en México. Cadernos de Pesquisa, 38(134), 503-533. Recuperado de http://www.scielo.br/pdf/cp/v38n134/ a1238134.pdf

Neuhauser, L. y Pohl, C. (2015). Integrating Transdisciplinarity and Translational Concepts and Methods into Graduate Education. En P. Gibbs (ed.), Transdisciplinary Professional Learning and Practice (pp. 99-120). Bern: Springer.

Nicolescu, B. (1999). The transdisciplinary evolution of learning. Recuperado de http://www.learndev.org/dl/nicolescu_f.pdf

Olivé, L. (2007). La ciencia y la tecnología en la sociedad del conocimiento. Ética, política y epistemología. México: FCE.

Palaiologou, I. (2010). The death of a discipline or the birth of a transdiscipline: subverting questions of disciplinarity within Education Studies undergraduate courses. Educational Studies, 36(3), 269-282. Recuperado de https://www.tandfonline.com/doi/ abs/10.1080/03055690903220180 
Piaget, J.; Lazarsfeld, P. y Mackenzie, W. M. (1973). Tendencias en la investigación en las ciencias sociales. Madrid: Alianza/ UNESCO.

Piaget, J. y H. Desmet. (1992). Epistemología e instrumentación en ciencias humanas. Barcelona: Herder.

Puiggrós, A. y R. Marengo. (2013). Pedagogías: reflexiones y debates. Buenos Aires: Universidad Nacional de Quilmes.

Ubal, M. y Píriz, S. (2009). ¿De qué hablamos cuando decimos pedagogía? Recuperado de http://www.inau.gub.uy/biblioteca/ piriz\%20ubal.pdf

Valladares, L. (2017). La "práctica educativa" y su relevancia como unidad de análisis ontológico, epistemológico y sociohistórico en el campo de la educación y la Pedagogía. Perfiles Educativos, XXXIX(158), 186-203. Recuperado de http:/www.redalyc.org/ pdf/132/13253901011.pdf

Velázquez, I. (2005). Una aproximación al mapa disciplinar de la pedagogía. Revista Iberoamericana de Educación, 35, 1-15. Recuperado de www.rieoei.org/deloslectores/811Velazquez.pdf 